\title{
Free Energy, Precision and Learning: The Role of Cholinergic Neuromodulation
}

\author{
Rosalyn J. Moran, ${ }^{1,2}$ Pablo Campo, ${ }^{1,3}$ Mkael Symmonds, ${ }^{1}$ Klaas E. Stephan, ${ }^{1,4,5}$ Raymond J. Dolan, ${ }^{1}$ and Karl J. Friston ${ }^{1}$ \\ ${ }^{1}$ Wellcome Trust Centre for Neuroimaging, Institute of Neurology, University College London, London WC1N 3BG, United Kingdom, ${ }^{2}$ Virginia Tech \\ Carilion Research Institute and Bradley Department of Electrical and Computer Engineering, Virginia Tech, Roanoke, Virginia 24016, ${ }^{3}$ Department of Basic \\ Psychology, Autonoma University of Madrid, 28049 Madrid, Spain, ${ }^{4}$ Translational Neuromodeling Unit, Institute for Biomedical Engineering, University of \\ Zurich and Swiss Federal Institute of Technology (ETH Zurich), 8092 Zurich, Switzerland, and ${ }^{5}$ Laboratory for Social and Neural Systems Research, \\ Department of Economics, University of Zurich, CH-8006 Zurich, Switzerland
}

Acetylcholine $(\mathrm{ACh})$ is a neuromodulatory transmitter implicated in perception and learning under uncertainty. This study combined computational simulations and pharmaco-electroencephalography in humans, to test a formulation of perceptual inference based upon the free energy principle. This formulation suggests that ACh enhances the precision of bottom-up synaptic transmission in cortical hierarchies by optimizing the gain of supragranular pyramidal cells. Simulations of a mismatch negativity paradigm predicted a rapid trial-by-trial suppression of evoked sensory prediction error (PE) responses that is attenuated by cholinergic neuromodulation. We confirmed this prediction empirically with a placebo-controlled study of cholinesterase inhibition. Furthermore, using dynamic causal modeling, we found that drug-induced differences in PE responses could be explained by gain modulation in supragranular pyramidal cells in primary sensory cortex. This suggests that ACh adaptively enhances sensory precision by boosting bottom-up signaling when stimuli are predictable, enabling the brain to respond optimally under different levels of environmental uncertainty.

\section{Introduction}

The Helmholtzian notion of the brain as a statistical inference machine (von Helmholtz, 1866) can be realized, under free energy formulations of perceptual inference, as a neurobiologically plausible implementation of predictive coding. In this framework, the brain entails a generative model of its environment that reproduces the hierarchical and dynamic generation of sensory input. Neuronal responses are proposed to reflect inference on the (hidden) causes of sensory stimuli that enable the brain to predict its sensations (Mumford, 1992; Rao and Ballard, 1999; Friston, 2010).

The implicit Bayes optimal recognition requires statistical distributions over hidden states and the environment to be encoded by neuronal activity in cortical hierarchies, where these representations are updated until they mirror real-world statistics through accurate predictions of sensory input. To weight hierar-

Received Sept. 4, 2012; revised Feb. 13, 2013; accepted March 20, 2013.

Author contributions: R.J.M., P.C., M.S., K.E.S., R.J.D., and K.J.F. designed research; R.J.M., P.C., M.S., and K.J.F. performed research; R.J.M. and K.J.F. contributed unpublished reagents/analytic tools; R.J.M. and K.J.F. analyzed data; R.J.M., P.C., M.S., K.E.S., R.J.D., and K.J.F. wrote the paper.

R.J.M. was supported by a Max Planck Award. K.J.F., R.J.D., and M.S. were supported by the Wellcome Trust. K.E.S. was supported by Neurochoice (SystemsX.ch) and an endowed ETH Chair from the René and Susanne Braginsky Foundation. P.C. was supported by a Ramon y Cajal Fellowship from the Spanish Ministry of Science and Innovation (RYC-2010-05748).

All the simulations reported in this paper are available in the DEM toolbox of SPM (http://www.fil.ion.ucl.ac. $\mathrm{uk} / \mathrm{spm} /$. The inversions can be simulated for different drug effects by changing DEM_demo_MMN.m \{Placebo: $M(1) \cdot h E=4 ; M(1) \cdot h C=\exp (-4)\}$ or $\{$ Galantamine: $M(1) \cdot h E=6 ; M(1) \cdot h C=\exp (-6)\}$.

Correspondence should be addressed to Rosalyn J. Moran, Virginia Tech Carilion Research Institute, 2 Riverside Circle, Roanoke, VA 24016. E-mail: rosalynj@vtc.vt.edu.

DOI:10.1523/JNEUROSCI.4255-12.2013

Copyright $\odot 2013$ the authors $\quad 0270-6474 / 13 / 338227-10 \$ 15.00 / 0$ chical predictions with greater or lesser importance, optimal inference assigns greater or lesser precision (inverse variance) to different hierarchical levels.

One candidate mechanism for signaling precision is neuromodulation: acetylcholine (ACh) is well equipped to influence low-level auditory processing through modulating postsynaptic gain. Traditional views of $\mathrm{ACh}$ as a nonspecific modulator of arousal that responds, through extrasynaptic volume transmission, to enhanced sensory and task demands (Giovannini et al., 2001; Hasselmo and McGaughy, 2004; Pepeu and Giovannini, 2004; Dani and Bertrand, 2007) are being rethought on the basis of improved (multiregion) microdialysis recordings in animal studies. These data suggest a selective cholinergic modulation of sensory cortex Nelson et al., 2005; Sarter et al., 2005; Benarroch, 2010; Fadel, 2011; Hasselmo and Sarter, 2011).

Under the free energy formulation (see Figure 1), ACh and other neuromodulators encode changes in the precision of (certainty about) prediction errors in sensory cortical hierarchies (Friston, 2008). Each level of a processing hierarchy sends predictions to the level below, which reciprocate bottom-up signals. These signals are prediction errors that report discrepancies between top-down predictions and representations at each level (Kiebel et al., 2009). This recurrent message passing continues until prediction errors are minimized throughout the hierarchy. The ensuing Bayes optimal perception rests on optimizing precision at each level of the hierarchy that is commensurate with the environmental statistics they represent. Put simply, to infer the causes of sensory input, the brain has to recognize when sensory information is noisy or uncertain and down weight it suitably in relation to top-down predictions (Yu and Dayan, 2005, Deco and 
Thiele, 2009). In this work, we address cholinergic neuromodulation as a candidate for optimizing precision. First we simulate (Bayes optimal) neural responses to sequences of repeated stimuli, under different levels of sensory precision (modulatory gain). We then compare simulated neuronal responses to empirical eventrelated responses from an electroencephalographic (EEG) study, using an oddball paradigm and a pharmacological (cholinergic) manipulation. The simulations and empirical results suggest that ACh biases perception toward bottom-up sensory processing by boosting prediction errors at the lower (sensory) levels of the hierarchy (Fig. 1). The empirical responses, and associated changes in postsynaptic gain, provide strong support for a specific role of ACh during perceptual inference under uncertainty.

\section{Materials and Methods}

Simulating brain responses using variational free energy minimization. Predictive coding schemes (Rao and Ballard, 1999) invoke recurrent neural message passing to simultaneously predict sensory stimuli and report prediction errors (e.g., to account for "pop-out," figuresurround responses in visual cortex (Lamme, 1995; Zipser et al., 1996). The free energy framework places predictive coding in a more general setting, using dynamic and hierarchical generative models of hidden environmental states (causes of sensory inputs) and an approximate posterior density on the states and parameters of the generative model, encoded by synaptic activity and weights, respectively. This approximate posterior (recognition) density is updated to maximize the evidence for the generative model (Kiebel et al., 2009). Mathematically, this optimization involves minimiz-

ing a variational free energy bound on log evidence (henceforth free energy) which, when minimized, provides a recognition density that approximates the true posterior-this is also known as approximate Bayesian inference. Crucially, the process of free energy minimization, in networks implementing predictive coding, can be used to predict the neuronal dynamics one would expect to measure using EEG (Figure 1; Friston and Kiebel, 2009). Here, we will use dynamic causal modeling (DCM) to test the theoretical assumptions about the neurobiological mechanisms that support Bayes optimal inference in an oddball task.

To produce quantitative predictions about this inference, we generated auditory stimuli in blocks of different frequencies. We then simulated how the brain would respond to the stimuli if it minimized free energy using a neuronally plausible predictive coding scheme. In brief, this scheme encodes beliefs about the world by associating synaptic activity with expected states of the world, causal structure (parameters controlling transitions among states) with synaptic connection strengths, and the precision (inverse variance) of random fluctuations of hidden states with synaptic gain (Friston, 2008). By assuming these beliefs are encoded with a Gaussian density, the expected states, parameters, and precision can be optimized in a fairly straightforward way using a gradient descent on free energy, which is also known as Bayesian filtering (cf., Kalman filtering). This Bayesian filtering or predictive coding can be formulated in terms of Bayesian updates that are driven by precisionweighted prediction errors. Mathematically, precision increases the influence of prediction errors, when they are precise. In neurobiological
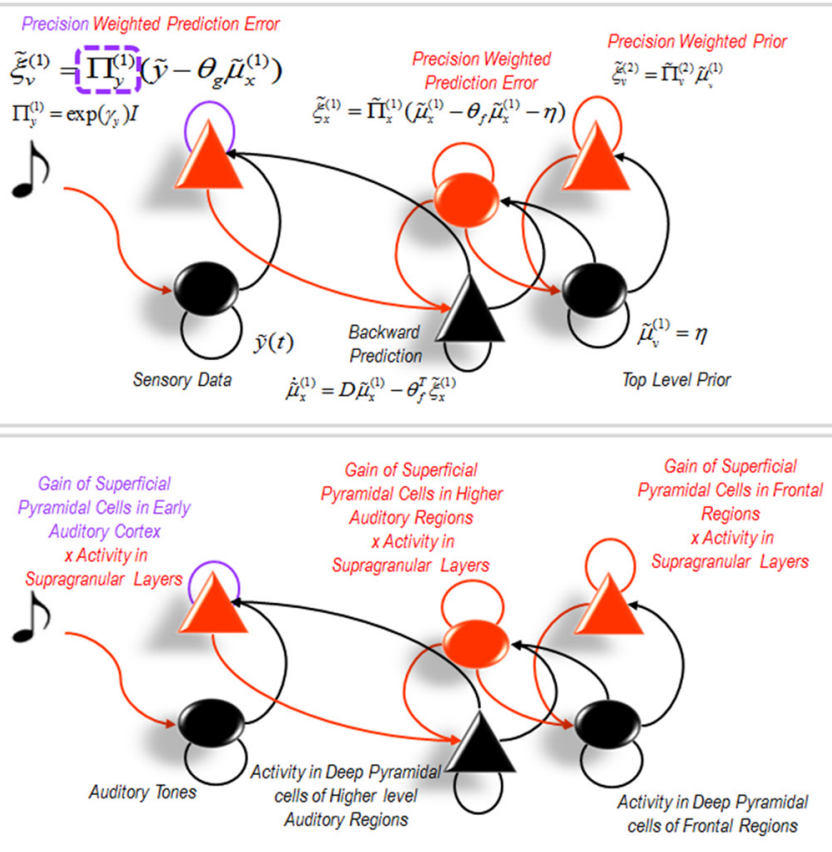

Figure 1. Simulating predictive coding through free energy minimization: algorithmic components and proposed neurobiological implemen(he time varying states and causes (Friston, 2008). Bottom, The ensuing Bayesian filtering or predictive functions of top-down and lateral predictions from the state units, which implies modulatory backward connections (e.g., mediated by theoretically in (Friston, 2008, 2009; Feldman and Friston, 2010). The DCM used in this paper distinguishes activity in two subpopulations of pyramidal cells (supragranular and infragranular) and their respective excitability or gain.

terms, it is generally assumed that prediction errors are encoded by pyramidal cells in the superficial layers of cortex that receive top-down predictions from deeper laminae in higher regions (Mumford, 1992; Cauller, 1995; Bar, 2003). The precision weighting of these prediction errors units depends on their postsynaptic gain (cf., Kalman gain). By changing the precision (synaptic gain) at a particular level of cortical hierarchy one can simulate the effects of manipulating cholinergic neuromodulation at this level, on the ensuing inference (Fig. 1).

In more detail, we assume that the hierarchical nature of cortical organization (Felleman and Van Essen, 1991) implies a hierarchical model of the sensorium that can be described by a set of stochastic differential equations of the following general form:

$$
\begin{aligned}
y & =g\left(x^{(1)}, v^{(1)}, \theta\right)+\omega_{v}^{(1)} \\
\dot{x}^{(1)} & =f\left(x^{(1)}, v^{(1)}, \theta\right)+\omega_{x}^{(1)} \\
& \vdots \\
v^{(i-1)} & =g\left(x^{(i)}, v^{(i)}, \theta\right)+\omega_{v}^{(i)} \\
\dot{x}^{(i)} & =f\left(x^{(i)}, v^{(i)}, \theta\right)+\omega_{x}^{(i)} \\
& \vdots \\
\omega_{x}^{(i)} & \sim N\left(0, \Pi_{x}^{(i)-1}\right) \\
\omega_{v}^{(i)} & \sim N\left(0, \Pi_{x}^{(i)-1}\right) \\
\Pi_{x}^{(i)} & =\exp \left(\gamma_{x}^{(i)}\right) \cdot I \\
\Pi_{v}^{(i)} & =\exp \left(\gamma_{v}^{(i)}\right) \cdot I
\end{aligned}
$$




\section{a The Roving Paradigm}

Switch to a deviant frequency
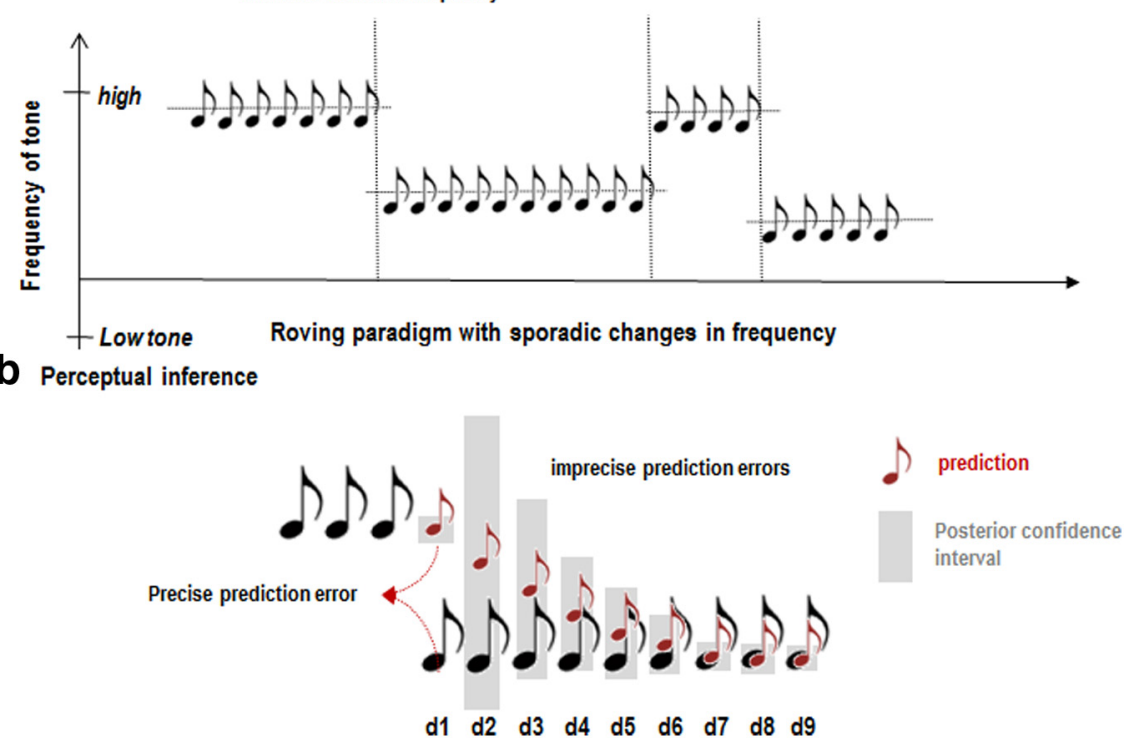

Figure 2. Paradigm and perception. This schematic illustrates the roving oddball paradigm ( $\boldsymbol{a}$ ) used in both the simulations and empirical study. After the presentation of a deviant tone, the expected precision of sensory information falls due to the profound prediction errors elicited by an unpredicted (deviant) stimulus, d1. With subsequent repetitions of the same stimulus, the stimulus becomes more predictable and confidence or precision recovers. Posterior confidence is illustrated with the gray bars in the bottom $(\boldsymbol{b})$ and is determined by the sensory precision expected. Crucially, eventrelated responses are assumed to report precision-weighted prediction errors. This means that the monotonically decreasing prediction error, with repetition, is modulated by precision to produce a characteristic MMN (precise prediction error) that almost disappears after presentation of the first deviant.

These equations model how sensory data of generated causes $v$, from level $i$ enter as inputs to lower levels, eliciting changes in hidden states at that level $x^{(i-1)}$. These hidden states then produce further hidden causes that cascade through lower hidden states to the bottom of the hierarchy that generates sensory input $y(t)$. These hidden variables (causes and states) could correspond to things like the motion of objects in the visual field. The functions $f$ and $g$ that determine how hidden variables conspire to produce sensations have parameters $\theta$ that represent causal structure in the world (Friston, 2005). These variables and their motion are subject to Gaussian random fluctuations $\omega^{(i)}$ with log-precision $\gamma^{(i)}$.

Given the form of the generative model (Eq. 1), one can write down the differential equations for predictive coding or Bayesian filtering in terms of prediction errors on the hidden variables.

$$
\begin{aligned}
& \dot{\tilde{\mu}}_{x}^{(i)}=D \tilde{\mu}_{x}^{(i)}+\frac{\partial \tilde{g}^{(i)}}{\partial \tilde{\mu}_{x}^{(i)}} \cdot \Pi_{v}^{(i)} \tilde{\varepsilon}_{v}^{(i)} \\
& \dot{\tilde{\mu}}_{x}^{(i)}=\quad+\frac{\partial \tilde{f}^{(i)}}{\partial \tilde{\mu}_{x}^{(i)}} \cdot \Pi_{x}^{(i)} \tilde{\boldsymbol{\varepsilon}}_{x}^{(i)}-D \cdot \Pi_{x}^{(i)} \tilde{\boldsymbol{\varepsilon}}_{x}^{(i)} \\
& D \tilde{\mu}_{v}^{(i)}+\frac{\partial \tilde{g}^{(i)}}{\partial \tilde{\mu}_{v}^{(i)}} \cdot \Pi_{v}^{(i)} \tilde{\varepsilon}_{v}^{(i)} \\
& \begin{aligned}
\dot{\tilde{\mu}}_{v}^{(i)}= & +\frac{\partial \tilde{f}^{(i)^{T}}}{\partial \tilde{\mu}_{v}^{(i)}} \cdot \Pi_{x}^{(i)} \tilde{\boldsymbol{\varepsilon}}_{x}^{(i)}-\Pi_{v}^{(i+1)} \tilde{\boldsymbol{\varepsilon}}_{v}^{(i+1)}
\end{aligned} \\
& \tilde{\varepsilon}_{x}^{(i)}=D \tilde{\mu}_{x}^{(i)}-\tilde{f}^{(i)}\left(\tilde{\mu}_{x}^{(i)}, \tilde{\mu}_{v}^{(i)}\right) \\
& \tilde{\varepsilon}_{v}^{(i)}=\tilde{\mu}_{v}^{(i-1)}-\tilde{g}^{(i)}\left(\tilde{\mu}_{x}^{(i)}, \tilde{\mu}_{v}^{(i)}\right)
\end{aligned}
$$

Equation 2 can be derived fairly easily by computing the free energy for the hierarchical model in Equation 1 and, using its gradients, perform a generalized descent. In neural network terms, Equation 2 says that error units $\tilde{\varepsilon}^{(i)}$ receive predictions from the same hierarchical level $\tilde{\mu}_{v}^{(i-1)}$ and the level above $\tilde{\mu}_{v}^{(i)}$. Conversely, conditional expectations (encoded by the activity of state units) are driven by prediction errors from the same level $\tilde{\varepsilon}_{v}^{(i+1)}$ and the level below $\tilde{\varepsilon}_{v}^{(i)}$. This is the essence of recurrent message passing between hierarchical levels to suppress free energy or prediction predictions. error (Friston (2008)). Equation 2 shows how precision $\Pi^{(i)}$ plays an important role in weighting the influence of prediction errors at any level of the hierarchy. In other words, by changing the precision on the prediction errors, we can bias inference toward sensory information or top-down (empirical) prior beliefs.

To simulate perceptual inference one simply integrates Equation 2, to see how the predictions and prediction errors respond to sensory input. To simulate an oddball paradigm, we generated a stream of auditory tones according to a roving design (cf., Haenschel et al., 2005; Fig. 2). This comprised mini-blocks of pure tones (up to 10 repetitions in length). The onset of a tone is caused by a Gaussian bump function that peaked at $100 \mathrm{~ms}$. This hidden cause $\left(v^{(1)}\right)$ perturbed two dynamic hidden states $x^{(1)}=\left[x_{1}^{(1)}, x_{2}^{(1)}\right]^{T}$ controlling the amplitude and frequency of auditory stimuli. The hidden states were mixed using parameters $\theta_{f}$ to drive their motion and were mapped, using parameters $\theta_{g}$ to the amplitude and frequency of auditory stimuli. This model was used to simulate tones of different frequencies (by changing the parameter $C$ in Eq. 3). We simulated a change from a high tone to a low tone, with 10 repetitions $(\mathrm{d} 1-\mathrm{d} 10)$. State noise was assumed to be zero mean with a log-precision of 16. This generative model can be written in the form of Equation 1 as the following:

$$
\begin{aligned}
y(t) & =\theta_{g} x^{(1)}+\omega_{v}^{(1)} \\
\dot{x}^{(1)} & =\theta_{f} x^{(1)}+\left[\begin{array}{c}
v^{(1)} \\
0
\end{array}\right]+\omega_{x}^{(1)} \\
v^{(1)} & =\eta(t)+\omega_{v}^{(2)} \\
\theta_{g} & =\left[\begin{array}{ll}
0 & 1 \\
C & 0
\end{array}\right] \quad \theta_{f}=\left[\begin{array}{cc}
-1 / 16 & 4 / 16 \\
-2 / 16 & -1 / 16
\end{array}\right]
\end{aligned}
$$

To simulate neuronal responses, we assumed that the brain was equipped with the same hierarchical model used to generate the stimuli, but did not know the parameter controlling frequency modulation over peristimulus time (the $C$ parameter in Eq. 3). The brain must therefore learn this parameter over successive repetitions. This learning also corresponds to a gradient descent on free energy and, under the simple linear model above, reduces to associative plasticity (Friston, 2008). Using the generative model in Equation 3, we simulated neuronal responses using Equation 2. This effectively updates posterior beliefs about the current stimulus using prediction errors (Fig. 1). Crucially, these Bayesian updates involve not only the parameters responsible for generating sensory predictions but also the precision encoding the uncertainty about those

We used a veridical prior for the presentation of a high-frequency tone $(C=4)$, then presented 10 tones at a frequency using $C=1(\mathrm{~d} 1-\mathrm{d} 10)$. After the scheme had observed each tone, the prior expectation about the unknown parameter was updated to the posterior expectation, similarly for the priors on the precision of sensory noise. We simulated the neuronal responses using the precision-weighted prediction errors of tone frequency (Fig. 1, $\left(\tilde{\xi}_{v}^{(1)}=\Pi_{v}^{(i)} \tilde{\varepsilon}_{v}^{(i)}\right.$,top). Assuming that prediction errors are reported by superficial pyramidal cells, which are the principal contributors to electrophysiological measurements, we can treat the precision-weighted prediction errors as event-related potentials (ERPs).

To model a placebo condition we used a Gaussian prior on the sensory log-precision (Fig. 1, $\Pi_{v}^{(1)}=\exp (\gamma) \cdot I$ ), with $p(\gamma)=N\left(\mu_{t-1}\right.$, exp $(-4)$ ), where $\mu_{t-1}$ is the posterior expectation of the sensory logprecision following the previous trial (starting with a log-precision of 4). To model the effects of galantamine we used a prior with a higher mean and log-precision: $p(\gamma)=N\left(\mu_{t-1}+2, \exp (-(4+2))\right)$. In other 
words, we assumed that galantamine increases both the brain's prior expectation about the precision of sensory input and the confidence about that expectation (Fig. 1, top). In terms of the underlying neurobiology, we propose that this increase in sensory precision is mediated by an associated neuromodulatory gain effect (Fig. 1, bottom), where greater ACh levels enhance postsynaptic responses in the superficial layers of primary auditory cortex.

As a point of comparison for the empirical data, we also simulated two alternative ways in which galantamine might affect sensory precision. The first "straw-man" alternative assumed galantamine reduced sensory precision; i.e., $p(\gamma)=N\left(\mu_{t-1}-2\right.$, $\left.\exp (-(4-2))\right)$. The second alternative assumed that galantamine had no effect on precision updating. In this case, we assumed that the log-precision remained at its initial value, i.e., $p(\gamma)=N(4, \exp (-4))$. Having produced predictions for ERPs in this oddball paradigm, under different assumptions about the effect of galantamine, we then measured real ERPs using the same paradigm (Fig. $3 a, b)$.

Subjects and pharmacological manipulation. We studied 13 righthanded, healthy volunteers (five female; aged $25 \pm 7$ years), using a within-subject crossover placebo-controlled double-blind design. Subjects attended on two sessions, exactly 1 week apart. Before drug administration, a baseline electrocardiogram (ECG) was performed to exclude cardiac conduction abnormalities. One hour before the EEG study, subjects were given either a tablet containing $8 \mathrm{mg}$ of galantamine or a multivitamin placebo. Tasks began $\sim 45$ min after EEG preparation. Galantamine increases the availability of ACh in cholinergic synapses by competitive inhibition of acetylcholinesterase, the enzyme responsible for its breakdown. Galantamine also enhances cholinergic neurotransmission by sensitizing nicotinic receptors (it both increases the probability of channel opening induced by $\mathrm{ACh}$ and slows down receptor desensitization) (Coyle and Kershaw, 2001; Samochocki et al., 2003). Following an oral dose, the peak plasma concentration is attained within $2 \mathrm{~h}$ and declines with a half-life of about $7 \mathrm{~h}$ (Huang et al., 2002).

Subjects were paid for their participation and consented to all procedures, which were conducted in accordance with the Declaration of Helsinki (1991) and approved by the local ethics committee.

Task and EEG acquisition. EEG recordings were made in a quiet dimly lit room using a 128-channel Biosemi system. Electrical signals were digitized at a sampling rate of $512 \mathrm{~Hz}$. Auditory stimuli were presented binaurally over headphones. The stimuli comprised a structured sequence of pure sinusoidal tones, with a roving or sporadically changing frequency (Fig. 2). This roving oddball paradigm (Garrido et al., 2008) comprises mini-blocks of 6-10 tone repetitions at frequencies of $500 \mathrm{~Hz}$, $550 \mathrm{~Hz}, 600 \mathrm{~Hz}, 650 \mathrm{~Hz}, 700 \mathrm{~Hz}, 750 \mathrm{~Hz}$, or $800 \mathrm{~Hz}$. At the end of each mini-block the frequency changed pseudorandomly to another frequency. The duration of each tone was $70 \mathrm{~ms}$, with $5 \mathrm{~ms}$ rise and fall times. On average, 180 stimuli for each repetition order were presented to each subject. Subjects were instructed to fixate on a central cross presented on a computer screen and perform an incidental visual task during which they were required to make a button press whenever the central fixation cross changed from gray to white or white to gray. This occurred pseudorandomly every $2-5 \mathrm{~s}$. Subjects were not asked to attend to the auditory stimuli, since this type of auditory oddball paradigm elicits mismatch potentials that are automatic or pre-attentive (Garrido et al., 2009a; Näätänen et al., 2010).

Data preprocessing and statistical analysis. EEG data were epoched offline to obtain $400 \mathrm{~ms}$ epochs corresponding to -100 to $300 \mathrm{~ms}$ peristimulus time. The epoched data were bandpass filtered from $1-40 \mathrm{~Hz}$, downsampled to $200 \mathrm{~Hz}$, and re-referenced to the nasion. The data were corrected for artifacts by thresholding (at $100 \mathrm{mV}$ ) and averaged according to repetition position in the mini-block. The first presentation, $\mathrm{d} 1$, (the deviant) the second presentation, $\mathrm{d} 2$, and so on, to the last possible presentation, d10, (the standard) were averaged over stimulus frequency. The event-related responses were subsequently baseline corrected (the analysis routines used for the present study are available in the academic freeware SPM8; http://www.fil.ion.ucl.ac.uk/spm/).

For our sensor level analysis, one electrode was selected from a frontocentral region channel C21; Garrido et al., 2007b). ERPs corresponding to trials $\mathrm{d} 1, \mathrm{~d} 2$, and $\mathrm{d} 10$ were averaged over subjects for each drug con- dition (Fig. 3c) and plotted with the SEM. A mismatch negativity (MMN) was seen in the grand-averaged data, most prominently in trial $\mathrm{d} 1$ at 150 $\mathrm{ms}$ for both placebo and galantamine conditions (Fig. $3 d$ ). The repetition suppression of the MMN was characterized with a repeated-measures ANOVA, using the mean evoked response from 140 to $160 \mathrm{~ms}$ as the repeated measure.

Source reconstruction. To define the prior location of electromagnetic sources for a subsequent DCM analysis, multiple sparse priors were used to estimate the cortical source of the MMN (Friston et al., 2008). A tessellated cortical mesh template surface in canonical Montreal Neurological Institute (MNI) anatomical space (http://www.bic.mni.mcgill. $\mathrm{ca} /$ brainweb) as implemented in SPM8, served as a brain model to estimate the current source distribution (Mattout et al., 2007). This dipole mesh was used to calculate the forward solution using a spherical head model. The inverse solution was calculated for deviant (d1) and standard (d10) trials for conditions of placebo and galantamine separately. Multiple sparse priors employ several hundred patches of activation that are iteratively reduced until an optimal number and location of active patches are found, using a greedy Bayesian search (Friston et al., 2008). Source activity measures were then interpolated into MNI voxel space and analyzed using statistical parametric mapping, at the betweensubject level, in the usual way (Kilner et al., 2005): a contrast of evoked signal strength, for standard relative to deviant auditory responses (d10d1) was computed for the placebo condition. This simple main effect of repetition (deviance) was computed at $p<0.05$ uncorrected using a two-sample $t$ test across subjects and used as an inclusive mask (the MMN mask) for a test of the orthogonal interaction between repetition and drug condition $\left([\mathrm{d} 10-\mathrm{d} 1]_{\text {placebo }}-[\mathrm{d} 10-\mathrm{d} 1]_{\text {galantamine }}\right)$. Note that we report uncorrected results at $p<0.05$ because these analyses only served to identify candidate sources in the subsequent DCM analysis. We observed a significant drug effect within the MMN mask in the middle temporal gyri bilaterally (Fig. $4 b$ ).

DCM. In DCM, ERPs are modeled as the response of a dynamic inputoutput system to exogenous (experimental) inputs (David et al., 2006; Kiebel et al., 2006). The DCM generates a predicted ERP as the response of a network of coupled sources to sensory (thalamic) input, where each source corresponds to a neural mass model of several neuronal subpopulations. For each source, we used a model based on the canonical microcircuit (CMC; Figure 4c; http://www.fil.ion.ucl.ac.uk/spm/). This ensemble model is based on a mesoscopic circuit description of cat primary visual cortex, which represents the cardinal features of functional macrocolumns throughout the cortical sheet (Douglas and Martin, 2004; Bastos et al., 2012). Each source includes four neuronal subpopulations: infragranular and supragranular pyramidal cell populations, a granular layer of stellate cells, and an inhibitory interneuron population. Each subpopulation has its own (intrinsic) dynamics modeled by standard neural mass equations. The sources and their interactions are fully specified by these equations that are formally related to neural mass models in the simulation literature (Breakspear et al., 2006; Rodrigues et al., 2010). The differential equations describe the evolution of hidden neuronal states (membrane potentials and currents) in the subpopulations comprising each source. The form of these equations can be found in previous papers (David et al., 2006; Garrido et al., 2007a, b; 2009b; Kiebel et al., 2008). In brief, a convolution operation transforms the average density of presynaptic input arriving at the population into an average postsynaptic membrane potential; in turn, a sigmoidal function transforms the average membrane potential of the population into its average firing rate. The sensitivity or gain of each subpopulation to its afferents is controlled by a lumped parameter, which induces self-inhibition, and this parameter is a lumped representation of the effect of several currents, including voltage-gated potassium currents, calcium-gated potassium channels, and slow recovery from inactivation of the fast sodium current. These currents cause the firing rate to fall for a given membrane potential mediating a reduction in postsynaptic gain.

These models have been validated using animal electrophysiological recordings (Moran et al., 2008, 2011). Crucially the parameters of the model can be inferred from empirical data, using standard Bayesian inversion techniques (Kiebel at al., 2006). This involves supplementing the neuronal model with a conventional electromagnetic forward model to 
a Free Energy Minimisation: Simulated EEG Responses Simulated ERP Placebo

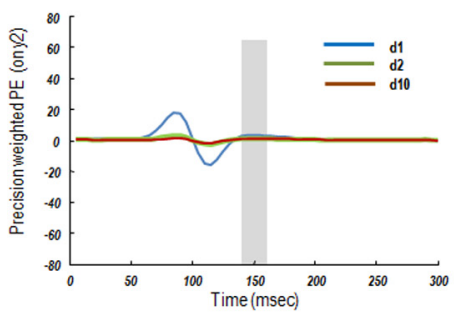

b

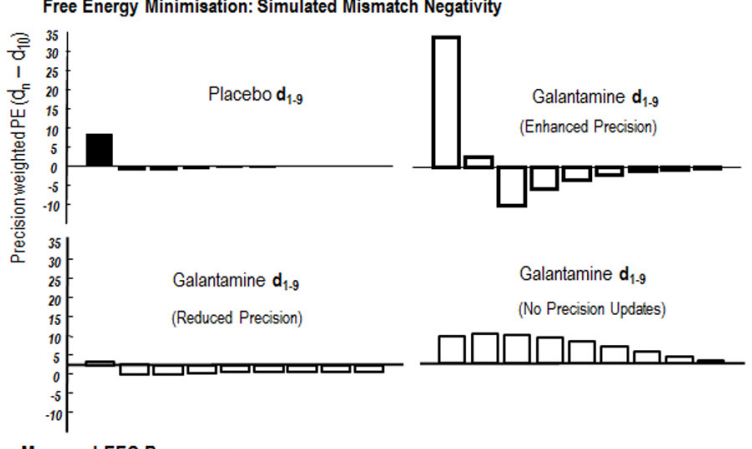

C Measured EEG Responses

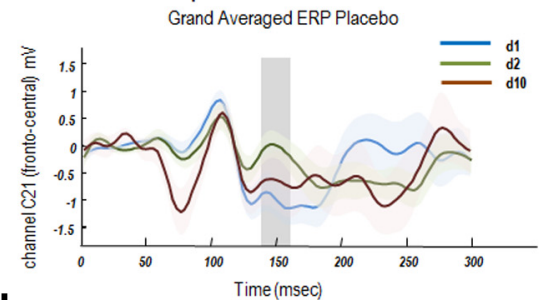

d Measured Mismatch Negativity
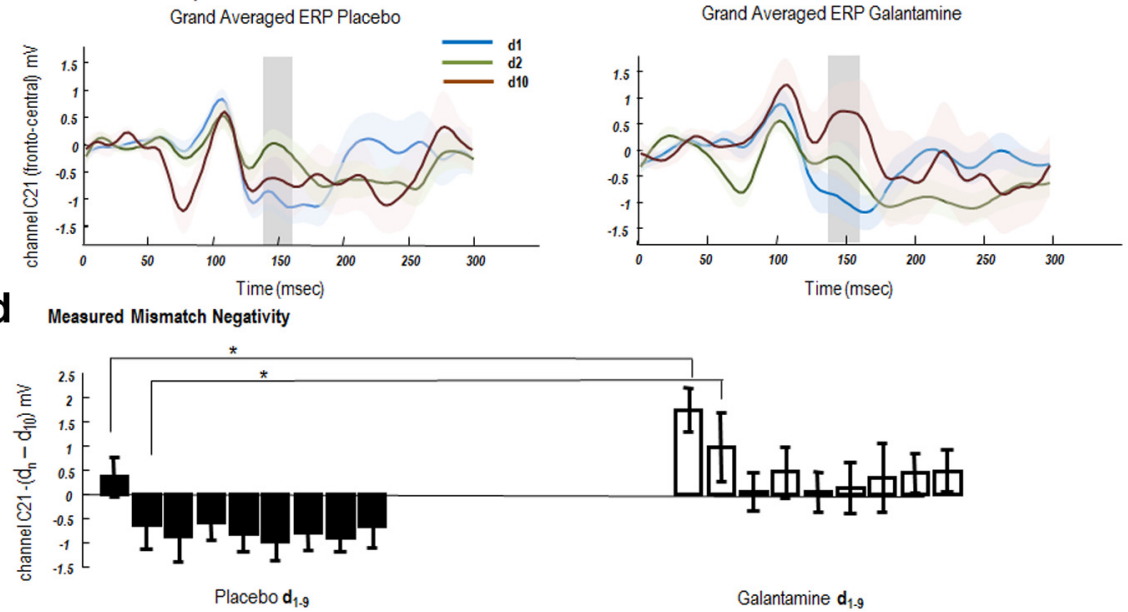

Figure 3. EEG responses: simulated and measured. $\boldsymbol{a}$, Simulated ERPs evoked by tones whose frequency deviates from preceding tones (d1) and are repeated until the tenth presentation (d10). These ERPs are the precision-weighted prediction errors (Fig. 1) of sensory data encoding the frequency of the tone $\left(y_{2}\right)$. Left, Simulated precision-weighted prediction error under placebo (d1, blue; $d 2$, green; $d 10$, red). The agent learns the tone frequency over successive repetitions, resulting in a reduction in the size of the evoked responses. Right, A similar profile is observed under galantamine, with elevated priors on sensory precision. Evoked responses are higher in magnitude and sustained for longer (d2). $\boldsymbol{b}$, Top, Simulated mismatch response (d10 $-\mathrm{dn}$ ), where d10 is set as the standard and $d 1-d 9$, a parametric deviant. The MMN is taken from the ERPs illustrated in $\boldsymbol{a}$. It is simply the difference between simulated evoked responses (precision-weighted prediction errors) between standards and deviants summed from 140 to $160 \mathrm{~ms}$ (shaded areas). Left, The placebo MMN shows a rapid one-shot learning, with a smaller MMN on d1 and a reversal in MMN polarity on $\mathrm{d} 2$, which returns to close to 0 at $\mathrm{d} 9$. Right, Galantamine MMNs are prolonged and have greater magnitude for all trials. Bottom, Two alternate MMN effects under different galantamine models (left) where galantamine reduced sensory precision and (right) where galantamine prevents precision updating over trials. c, Scalp EEG measurements of auditory evoked potentials. Grand-averaged waveforms from a single frontocentral electrode (C21) for the presentation of the first deviant tone in a sequence (d1), second tone (d2), and final tone (d10), averaged across tones of all frequencies, under placebo (left) and galantamine (right). MMN effects are evident in both drug conditions $\sim 150 \mathrm{~ms}$. $\boldsymbol{d}$, The MMN effect across all nine tone repetitions (d10 $-\mathrm{dn})$. The MMN effect was significantly different across repetitions and drug state. In particular both $\mathrm{d} 1 \mathrm{and} \mathrm{d} 2$ induced MMNs were greater on galantamine than on placebo.

map source activity to sensors (Kiebel et al., 2006). Given priors over the source locations, noninvasive ERPs can then be used to estimate the parameters of the neuronal model (Garrido et al., 2007b). This enables one to test, using pharmacological manipulations, which synaptic or coupling parameters are affected when changing the levels of specific neurotransmitters, such as increasing the level of ACh with galantamine in this study. Extrinsic connections in these models follow the same rules on which predictive coding schemes are based: forward connections from superficial pyramidal cells synapse on granular layer IV and deep cell layers, while backward connections from deep pyramidal cells terminate outside layer IV (Felleman and Van Essen, 1991).

This CMC model (Fig. 4c) was used to model explicitly the laminar specificity of forward and backward connections that are central to predictive coding, where forward connections convey prediction errors to higher levels and backward connections send predictions back to lower areas. The CMC thus allows us to test formal hypotheses, or models, regarding the effects of galantamine; namely, the drug could alter (1) the influence of prediction errors on their targets; instantiated as a change in forward connection strengths; or (2) the precision of prediction errors; instantiated as a change in the gain of superficial pyramidal cells encoding prediction errors, or (3) the influence of predictions, instantiated as a change in the strength of backward connections. We specified a model space along these dimensions, resulting in a model space with 10 competing hypotheses (Fig. $5 a$ ), each of which could potentially explain the differences in the ERP to the largest oddball response (d1) for galantamine relative to placebo. In comparing these DCMs, using Bayesian model comparison, we hoped to show that galantamine increased the gain of superficial pyramidal cells early in the auditory hierarchy.

For computational expediency, DCMs were fitted to sensor data following dimensionality reduction to eight channel mixtures or spatial modes. These were the eight principal modes of a singular value decomposition of the channel data between 0 and $250 \mathrm{~ms}$, over trial types of interest. Interestingly, DCMs are inverted using exactly the same variational scheme (variational Laplace) that underlies predictive coding (Kiebel et al., 2008). After model inversion or optimization, the variational free energy can then used as an approximate log model evidence for Bayesian model comparison.

\section{Results}

In brief, we found a remarkable correspondence between simulations of Bayes optimal sensory learning and the empirical responses at the level of scalp EEG and within the network of sources generating EEG signals. In what follows, we describe simulated (Bayes optimal) responses that, we suppose, underlie $\mathrm{MMN}$ responses elicited during the roving auditory oddball paradigm (Haenschel et al., 2005). We simulated EEG responses to unexpected or deviant auditory tones that gradually become "standard" tones, under placebo and galantamine. We then report the empirical findings, in light of the simulations, focusing on the repetition suppression of responses over time, in sensor space, and the underlying synaptic mechanisms, in source space.

\section{Simulating perception under placebo and galantamine}

To simulate the processing of auditory stimuli in the roving oddball paradigm, we first generated auditory stimuli, using a model 
of the auditory environment with two levels of causal dynamics (Eq. 3). We then applied Bayesian filtering (Eq. 2) to these sensory data and harvested the precisionweighted prediction errors generated over peristimulus time, for successive tone presentations.

Figure $3 a$, left, shows the (precisionweighted) prediction error in the placebo condition for the first deviant. A large precision-weighted prediction error is observed with a late deflection at around 150 ms (blue curve). This almost disappears on the second trial (green curve), which has a form similar to $\mathrm{d} 10$ (red curve). In other words, the first deviant stimulus produces large amplitude prediction errors that are boosted by an inappropriately high precision, established by the preceding sequence of predictable standards. This produces the MMN. After the first deviant, sensory precision is reduced (by the high prediction errors in the previous trial), rendering subsequent prediction errors less precise and thereby attenuating neuronal responses. These simulation results suggest a type of oneshot learning or repetition suppression, where the brain responds to a new tone with a precise prediction error and then reduces the precision of subsequent errorrelated responses as precision is updated and new parameters of the generative model are learned. Under galantamine (Fig. $3 a$, right), the high a priori precision on the sensory prediction error increases the response elicited by the first presentation of a new tone (blue curve), and attenuates the reduction of precision on subsequent presentations leading to larger responses at the second deviant (green curve) and a more protracted repetition suppression of subsequent responses.

To quantify this repetition suppression, we summed the prediction error over 140-160 ms (where MMNs are typically observed) of peristimulus time for each trial and computed the difference for each repetition, compared with $\mathrm{d} 10$ (defined as the standard). These differences correspond to a simulated MMN with arbitrary units (a.u.) (Fig. 3b). For the placebo MMN, when comparing trial $\mathrm{d} 10$ and trial $\mathrm{d} 1$, we observe a MMN of 8.1 a.u. that reverses polarity and reduces in size to -1 a.u. on trial $\mathrm{d} 2$. The MMN effect then gradually increases again to baseline $(-0.1$ a.u.) for d9. In contrast, galantamine increases the amplitude of the MMN and slows its decline (Fig. $3 b$ ): comparing trial $\mathrm{d} 10$ and trial d1, we observe a difference of magnitude of 34 a.u., which remains positive at 2.5 a.u. on the subsequent trial, d2. The MMN effect decreases slowly to a baseline for $\mathrm{d}$ 9. It is this quantitative difference in the MMN at the first and subsequent repetitions of a deviant stimulus that we hoped to observe empirically:

Allowing galantamine to change precision in other ways produced very different response profiles. Our first alternate hypothesis was that galantamine reduced, rather than increased, sensory precision. This assumption resulted in a reduced $\mathrm{MMN}$ response
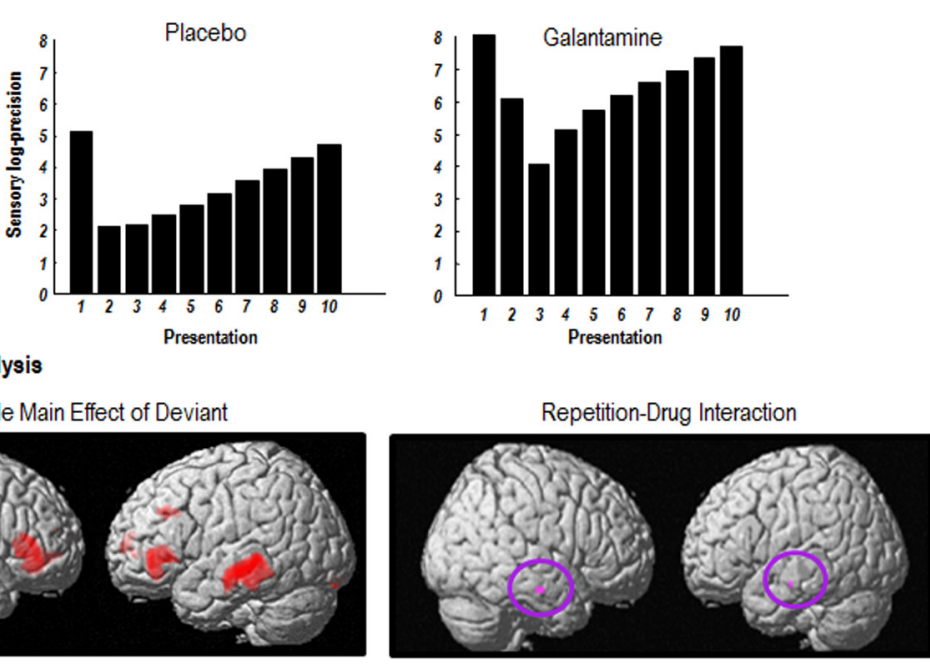

anonical Microcircuit in each source

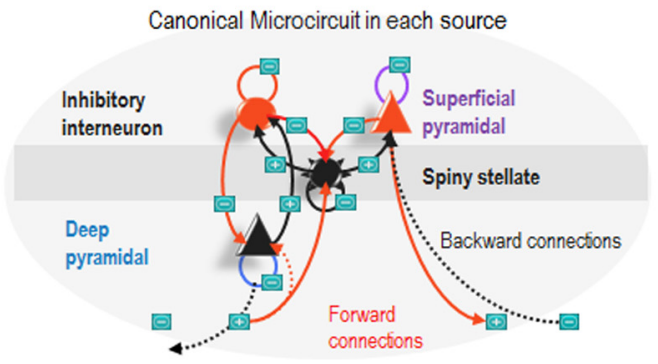

Figure 4. Synaptic effects: simulated and measured. $\boldsymbol{a}$, Simulated changes in precision parameter estimates. Left, The updates to precision parameters that encode sensory log-precision exhibit a phasic learning profile, with high values on the first deviant

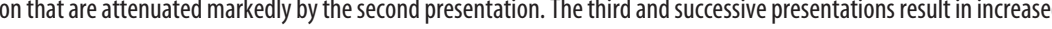
encoded with higher certainty and exhibit a different optimization, whereby high precision during the first oddball drops on the onto a canonical cortical surface and thresholded at $p<0.05$ uncorrected. The local maxima in frontal vertices were used as prior locations in the DCM analysis. The image served as a mask on tests for drug $\times$ repetition interactions. Right, Bilateral tions among subpopulations within each source in the neural mass model. Purple and blue highlight the intrinsic connections were modulated by galantamine in the DCM reported in the next figure.

of 2.9 a.u. $(\mathrm{d} 10-\mathrm{d} 1)$, which reduces polarity on $\mathrm{d} 2$ to -0.5 . Our null hypothesis was that galantamine precluded precision updates. This produced a very different profile, driven by slower changes in extrinsic connections that underlie learning. Here the MMN on the second trial d2 (7.2 a.u.) was greater than on d1 (6.7 a.u.).

\section{Empirical evoked brain responses and the MMN under placebo and galantamine}

To compare our simulation-based predictions to empirical MMN responses, we examined sensor-level evoked responses. We selected a frontocentral electrode (channel C21) typically found to express the MMN and computed the grand-averaged responses for the placebo and galantamine conditions (Fig. 3c). These responses exhibited the same form of repetition suppression that we saw in the simulations, with trial $\mathrm{d} 2$ (Fig. $1 c$, green curve) more similar to d10 (red curves) and a deflection at around $150 \mathrm{~ms}$ for the first oddball tone (blue curves). We used the MMN effect (between 140 and $160 \mathrm{~ms}$; defined here for the real data as the deviant standard) to test for statistical differences between the two drug conditions: 
a
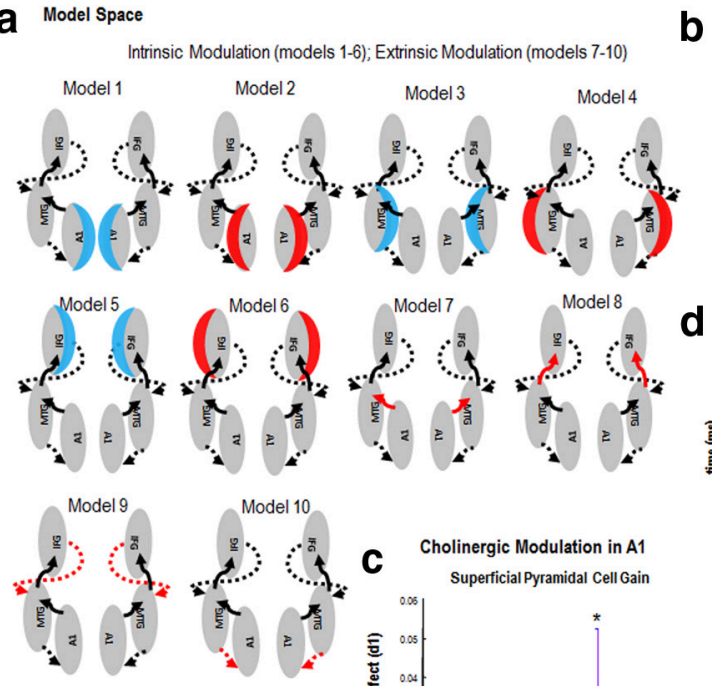

Forward Connection
Backward Connection

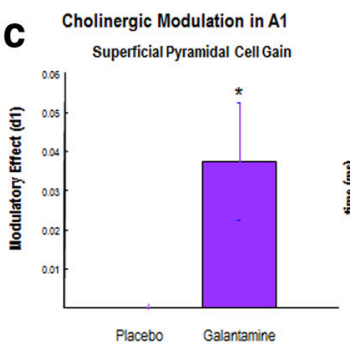

b

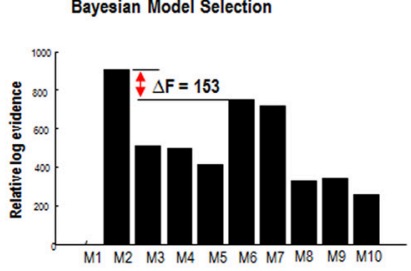

d
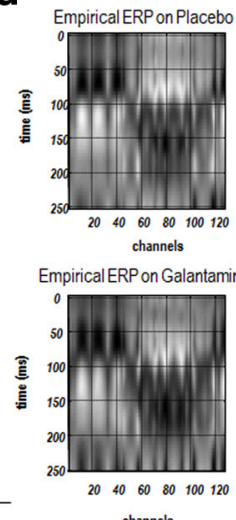

channels

Figure 5. Network modulation by galantamine. $\boldsymbol{a}$, Models tested to discover where galantamine boosted event-related responses. These modeled an effect of galantamine on either the gain of deep pyramidal cells (blue shading), the gain of superficial pyramidal cells (red shading), or extrinsic long-range connections (red arrows). $\boldsymbol{b}$, Bayesian model comparison revealed very strong evidence in favor of model 2 (compared with second best performing model). Model 2 models the effects of galantamine as a gain modulation of supragranular pyramidal cells in bilateral primary auditory cortex. $c$, The direction of the gain effect, under galantamine relative to placebo, shows enhanced gain at supragranular pyramidal cells with a posterior probability $=1$ (shown with 95\% Bayesian confidence intervals). $\boldsymbol{d}$, Fitting the DCM to empirical data shows a high correspondence across peristimulus time and channels for both placebo and galantamine responses.

As shown in Figure $3 c$, we observed an MMN in response to the onset of a deviant tone at a time commensurate with previous studies at $\sim 150 \mathrm{~ms}$. For each of the 13 subjects, we then quantified nine subject-specific MMNs (averaged over 140-160 ms) for each drug state by computing the difference between $\mathrm{d} 10$ and $\mathrm{d} 1$ to $\mathrm{d} 9$ (Fig. 3d) as in the simulations. We entered these differences into a repeated-measures ANOVA and found both a significant effect of repetition $(p<0.001)$ and a significant effect of drug $(p<0.05)$. Although we did not observe a significant drug $\times$ repetition interaction, we did find a significantly greater MMN under drug compared with placebo on the first and second presentation of a new tone ( $\mathrm{d} 1$ and $\mathrm{d} 2$ relative to $\mathrm{d} 10 ; p<0.05$, two-sample, one-tailed $t$ test; Fig. $3 d$ ). Furthermore, we observed an overall profile for the drug condition that is very similar to the simulated MMN when galantamine enhanced precision. Our empirical results show an enhanced MMN on galantamine at $\mathrm{d} 1$ compared with placebo that persists at $\mathrm{d} 2$, while the placebo MMN reverses in polarity after the first oddball (Fig. $3 d$ ). These empirical response profiles are inconsistent with either of the alternative models of galantamine effects (Fig. $3 b$ ).

\section{Optimal changes in synaptic efficacy and gain}

Under our biological implementation of Bayes optional perception, model parameters (like $C$ in Eq. 2) are encoded by the strength of extrinsic connections between brain regions; while log-precisions are encoded by the gain of prediction error units (i.e., intrinsic excitation of supragranular pyramidal cells). Our precision estimates show biphasic changes, where the initial oddball coincides with a high precision estimate $(\mathrm{d} 1=5.1$ a.u. $)$ that undergoes a large reduction $(\mathrm{d} 2=2.1$ a.u. $)$ and a subsequent

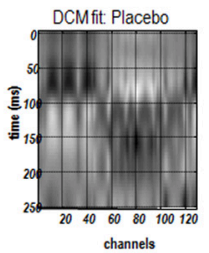

channels

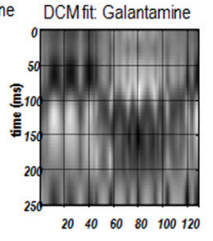

channels rebound (d10 $=4.7$ a.u.) (Figure $4 a$, left). From Figure $3 a$, we see that the corresponding evoked responses reflect these estimates of precision, because they are based on precision-weighted prediction errors. In the galantamine simulations (Fig. $4 a$, right), however, there was a profound difference in the log-precision updates, compared with placebo. The logprecision at the first deviant was higher (8.1 a.u.) and remained relatively high after the second tone repetition $(\mathrm{d} 2=$ 6.1 a.u.), reaching a minimum at $\mathrm{d} 3$ (Fig. $4 a$ ). This difference in the optimization of (cholinergic) precision or gain is reflected in the simulated evoked responses (Fig. 3a), with enhanced and prolonged precisionweighted prediction errors over repetitions, compared with placebo. Neurobiologically, this would correspond to a qualitative difference in the changes in gain of superficial pyramidal cells encoding prediction error, a prediction we assessed empirically using DCM:

\section{DCM results and their relationship to theoretical predictions}

In this section, we focus on the synaptic mechanisms producing the repetition suppression in sensor space described above. To address this, we model the generation of evoked responses in source space using DCM and parameterize this model in terms of coupling strengths and postsynaptic gain that have a direct interpretation in relation to the above simulations.

The locations of electromagnetic (dipolar) sources in our DCM were based on a contrast of source-localized ERPs that tested for drug effects. After source localization on a canonical mesh, we tested for a simple main effect of repetition $(\mathrm{d} 10-\mathrm{d} 1)$ in the placebo condition and found bilateral effects in middle temporal gyri (left MTG: $x=-66, y=-30, z=-4$; right MTG: $x=64, y=-16, z=-18$ ) and inferior frontal gyri (left IFG: $x=$ $-46, y=40, z=2$; right IFG: $x=36, y=48, z=-2$ ) (Fig. $4 b$ ). Using the results of this contrast as an inclusive mask, we then tested for an interaction of repetition and drug, identifying a significant interaction in MTG bilaterally (left MTG: $x=-60$, $y=-14, z=-18$, right MTG: $x=62, y=-14, z=-18$; Fig. $3 b$ ). We used the local maxima of the simple main effect in bilateral frontal cortex and the local maxima of the interaction in bilateral MTG as our prior source locations for the DCM. We also included primary auditory cortex bilaterally (left A1: $x=-42$, $y=-22, z=7$; right A1: $x=46, y=-14, z=8$ ), as per Garrido et al. (2008). This ensured that all levels of auditory network processing tones were included (Fig. 4). This three-level hierarchy is consistent with previous models of the MMN (Garrido et al., 2007a,b; 2008; 2009a,b). To test for the predicted differences in placebo and galantamine, we focused on the first presentation of the deviant (d1), because this is not confounded by sensory learning, under the different drug conditions, in subsequent repetitions. Our hypotheses (models) allowed for the galantamine to exert its effects at different locations in the predictive coding hierarchy: (1) forward connections from superficial pyramidal cells reporting prediction errors, (2) postsynaptic gain at super- 
ficial pyramidal cells encoding the precision of prediction errors, and (3) backward connections from deep pyramidal cells reporting predictions (Fig. 5a). For completeness we included models in which galantamine increased the gain of deep pyramidal cells (Fig. 5a, blue). In detail, we compared 10 models of grandaveraged ERPs (average seven subjects), where galantamine changed (bilaterally): the gain of deep pyramidal cells in primary auditory cortex (model 1), the gain of superficial pyramidal cells in primary auditory cortex (model 2), the gain of deep pyramidal cells in MTG (model 3), the gain of superficial pyramidal cells in MTG (model 4), the gain of deep pyramidal cells in IFG (model 5 ), the gain of superficial pyramidal cells in IFG (model 6), forward connections to MTG (model 7), forward connections to IFG (model 8), backward connections to MTG (model 9), and backward connections to primary auditory cortex (model 10) (Figure $5 a$ ). Figure $5 b$ shows the results of a Bayesian model comparison showing that the evidence for model 2 is greatest, with very strong evidence relative to the next best model, model 6 $(\log$ Bayes factor $=153)$. This means that the effect of galantamine can be best explained as a change in the gain of superficial pyramidal cells in primary auditory cortex. The direction of this effect is shown in Figure $5 c$ and suggests that galantamine enhances the gain in these bilateral sensory sources with a posterior probability of nearly $100 \%$. The fits to the empirical data under the best model are presented in Figure $5 d$.

In summary, there is a remarkable convergence between the predictions of Bayes optimal inference and learning under uncertainty and the empirical results obtained with a roving MMN paradigm. This convergence was both phenomenologically, in terms of repetition suppression at the sensor level, and mechanistically, in terms of underlying changes in synaptic gain at the source level (as inferred by DCM). These results are consistent with a key role for ACh in modulating the gain of superficial pyramidal cells in early sensory regions that may encode prediction error in predictive coding formulations of hierarchical inference.

\section{Discussion}

In this work, we have used a psychopharmacological study to test specific and formal predictions about the effect of cholinergic manipulations on event-related responses and their repetition suppression. These predictions rest on a Bayes optimal formulation of perceptual inference and learning that can be implemented in a neuronally plausible way. In brief, our results are consistent with a role for ACh in setting the gain of prediction error units implied by predictive coding in the brain. Neurobiologically, our DCMs represent postsynaptic gain via a parameter that determines the intrinsic excitability of a subpopulation (Moran et al., 2007). In predictive coding formulations of free energy minimization, this gain corresponds to the precision of prediction errors. By assigning ACh the role of signaling sensory precision, we have shown how its augmentation can reduce adaptation to surprising stimuli in sensory cortices. Our simulations and empirical results suggest that when stimuli are repeated in a predictable way, the statistical regularity leads to an adaptive increase in the expected precision of sensory prediction errors. This could be mediated (in part) by increased cholinergic neurotransmission that is suppressed after an unpredicted or surprising (deviant) stimulus. This withdrawal of a neuromodulatory boost leads to a rapid repetition suppression during successive presentations of the same (initially surprising and then increasingly predictable) stimulus. Several intracellular processes may underlie such repetition suppression, including slow after- hyperpolarizing currents $\left(\mathrm{sI}_{\mathrm{AHP}}\right)$ that are mediated by calciumdependent potassium channels and decrease neuronal excitability and firing rate (for a review, see Faber and Sah, 2003). Importantly, ACh modulates this process, e.g., by activation of muscarinic receptors, which suppress $\mathrm{sI}_{\mathrm{AHP}}$ through a cGMPdependent mechanism (Krause and Pedarzani, 2000). This activation may maintain a high postsynaptic gain and facilitate oscillatory activity, with a shift toward higher frequencies (desynchronization) in the power spectra of neuronal recordings (Liljenström and Hasselmo, 1995).

In summary, pharmacologically augmenting cholinergic neuromodulation appears to boost event-related responses to deviant stimuli and attenuate their subsequent repetition suppression. Using precision-weighted prediction errors from our Bayes optimal (predictive coding) scheme, we could predict suppression of the MMN response under the different drug conditions (Fig. 3). Moreover, DCM of a temporofrontal network suggested that the observed differences in event-related responses can be attributed to cholinergic gain modulation of supragranular pyramidal cells in early auditory cortex (Fig. 5). In short, these findings suggest that ACh mediates the representation of precision and acts to facilitate the bottom-up signaling of sensory information (through precise prediction errors). In the longterm this type of analysis may be applied to empirical data alone, to inform the computational consequences of pathophysiological changes. For example, if a DCM analysis reveals particular changes in directed extrinsic connections or intrinsic gain, one may be able to map this to changes in perceptual prediction error processing, e.g., in neuropsychiatric disorders such as schizophrenia (Marr, 2010; Adams et al., 2012).

Previous theoretical circuit level proposals have suggested that ACh supports learning and memory processes by switching the cortex from a "read-out " to "read-in " mode, providing an enhancement of sensory-evoked afferent responses and suppression of internal cortical processing (Hasselmo and McGaughy, 2004). This proposal is consistent with the current study. Microdialysis measures of ACh in behaving animals support this view, with increases observed in modality-specific sensory cortex and hippocampus during the acquisition of novel, behaviorally relevant stimuli. Indeed, it has been demonstrated in the auditory domain that a sensory "memory" relies upon cholinergic modulation (Miasnikov et al., 2008). Similarly, in human studies with cholinergic agonists, the specificity of perceptual learning for behaviorally relevant features is enhanced (Rokem and Silver, 2010), while validity effects in spatial cueing tasks are diminished (Bentley et al., 2004; Thiel and Fink, 2008; Vossel et al., 2008), indicative of selective boosting of stimulus-related information. Our analysis of the simulated and empirical responses recapitulates some of these established ideas about the role of cholinergic signaling in perception and attention. Our findings fit comfortably with previous theoretical and empirical accounts; namely, that ACh boosts bottom-up signals in response to uncertainty (Yu and Dayan, 2002, 2003; Bentley et al., 2004). In short, there is a wealth of theoretical and empirical evidence for a computational role of $\mathrm{ACh}$ in promoting the influence of sensory evidence in perception and attention, which our study supplements with a functional anatomy at the systems level.

One of the key features of the responses elicited by the roving paradigm is a repetition suppression, following a deviant stimulus, which recovers with subsequent presentations (Fig. 3). This profile has been reported previously in intrinsic (within-source) DCM connections exhibiting biphasic changes that were much faster than changes in extrinsic (between-source) connections 
(Garrido et al., 2009a). We extend these findings to show that this short-term plasticity may be modulated by ascending neuromodulatory systems. Previous research has shown that MMN responses are enhanced following cholinergic stimulation, particularly nicotinic stimulation in healthy controls (Baldeweg et al., 2006) and patient populations (Engeland et al., 2002). We simulated these electrophysiological responses by weighting prediction errors, during recognition dynamics, more highly under the influence of galantamine. This results in exaggerated and prolonged MMN-like responses over repetitions of a new stimulus. Crucially, this effect can be simulated easily, when sensory precision remains higher for longer periods.

Given the ability to characterize the putative neuronal implementation of predictive coding schemes using DCM (Garrido et al., 2008), we tested whether the sensitivity or gain of neuronal populations encoding prediction error was increased in human subjects by pharmacological enhancement of ACh. ACh has long been known to enhance the firing of cortical pyramidal neurons (Krnjevi and Phillis, 1963), inducing a decrease in spike frequency adaptation via several mechanisms, including the reduction of hyperpolarizing potassium currents (Benardo and Prince, 1982; Cole and Nicoll, 1984; Huang et al., 1993). This effect was modeled in our DCMs via a gain parameter that enhanced the excitability of supragranular pyramidal cells (through decreased self-inhibition).

Our DCM analysis helps to resolve previous ambiguities regarding the effects of ACh on top-down versus bottom-up processing in human neuroimaging experiments. Using standard univariate analyses, the neural network responsible for these, possibly reciprocal, adjustments under ACh has remained unclear (Thiel and Fink, 2008), given the difficulty of specifying how a bottom-up enhancement and top-down reduction would manifest in measurements of brain responses such as functional magnetic resonance imaging. Our DCM results suggest that under cholinesterase inhibition, sensory cortices respond to incoming stimuli with exaggerated and prolonged trial-by-trial responses at superficial pyramidal cells, which are the source of bottom-up or forward projections. This enhanced bottom-up effect is mediated via tonic increases in $\mathrm{ACh}$ in our simulations and experiments. It is possible that other brain regions, such as the prefrontal cortex or insula, modulate cholinergic afferents in a phasic fashion through inputs to the basal forebrain (Sarter et al., 2009). This would represent top-down control of facilitation of bottom-up inputs. This type of precision modulation, i.e., state-induced changes in sensory precision, can be included in generalized Bayesian schemes to provide a compelling metaphor for attentional modulation (Feldman and Friston, 2010).

In general, hierarchical inference using empirical Bayes, as implemented in our simulations, provides a mechanism by which neural circuits can selectively up weight and down weight particular features in the sensorium. When making perceptual inferences about the states of the world and their causes, the brain should up weight reliable sensory evidence by increasing the precision of signals from lower level cortical regions, e.g., primary sensory areas. For people familiar with statistical procedures this is nothing more than weighted least-squares, where noisy data are down weighted. The framework of Yu and Dayan (2002) proposes that ACh modulates uncertainty about high-level representations. We show here an equivalent and complementary effect, where ACh boosts bottom-up sensory evidence in the auditory hierarchy. The resulting adaptive sensory bias may be implemented via gain control in a manner suggested by previous physiological accounts of cholinergic modulation. Of course, our EEG measurements do not allow us to characterize the source of cholinergic modulation per se, e.g., between prefrontal regions and basal forebrain cholinergic neurons, which may serve as a route through which enhanced precision is initiated.

In summary, the proposition that neuromodulators encode the precision or predictability of environmental states is supported here by empirical evidence that sensory regions respond with enhanced gain under cholinergic enhancement, producing exaggerated and prolonged mismatch responses that localize to superficial pyramidal populations encoding prediction error (Mumford, 1992).

\section{References}

Adams RA, Perrinet LU Friston K (2012) Smooth pursuit and visual occlusion: active inference and oculomotor control in schizophrenia. PloS One 7:e47502. CrossRef Medline

Baldeweg T, Wong D, Stephan KE (2006) Nicotinic modulation of human auditory sensory memory: evidence from mismatch negativity potentials. Int J Psychophysiol 59:49-58. CrossRef Medline

Bar M (2003) A cortical mechanism for triggering top-down facilitation in visual object recognition. J Cogn Neurosci 15:600-609. CrossRef Medline

Bastos AM, Usrey WM, Adams RA, Mangun GR, Fries P, Friston KJ (2012) Canonical microcircuits for predictive coding. Neuron 76:695-711. CrossRef Medline

Benardo LS, Prince DA (1982) Cholinergic excitation of mammalian hippocampal pyramidal cells. Brain Res 249:315-331. CrossRef Medline

Benarroch EE (2010) Acetylcholine in the cerebral cortex. Neurology 75: 659-665. CrossRef Medline

Bentley P, Husain M, Dolan RJ (2004) Effects of cholinergic enhancement on visual stimulation, spatial attention, and spatial working memory. Neuron 41:969-982. CrossRef Medline

Breakspear M, Roberts JA, Terry JR, Rodrigues S, Mahant N, Robinson PA (2006) A unifying explanation of primary generalized seizures through nonlinear brain modeling and bifurcation analysis. Cereb Cortex 16: 1296-1313. Medline

Cauller L (1995) Layer I of primary sensory neocortex: where top-down converges upon bottom-up. Behav Brain Res 71:163-170. CrossRef Medline

Cole AE, Nicoll RA (1984) The pharmacology of cholinergic excitatory responses in hippocampal pyramidal cells. Brain Res 305:283-290. CrossRef Medline

Coyle J, Kershaw P (2001) Galantamine, a cholinesterase inhibitor that allosterically modulates nicotinic receptors: effects on the course of Alzheimer's disease. Biol Psychiatry 49:289-299. CrossRef Medline

Dani JA, Bertrand D (2007) Nicotinic acetylcholine receptors and nicotinic cholinergic mechanisms of the central nervous system."Annu Rev Pharmacol Toxicol 47:699-729.

David O, Kiebel SJ, Harrison LM, Mattout J, Kilner JM, Friston KJ (2006) Dynamic causal modeling of evoked responses in EEG and MEG. Neuroimage 30:1255-1272. CrossRef Medline

Deco G, Thiele A (2009) Attention-oscillations and neuropharmacology. Eur J Neurosci 30:347-354. CrossRef Medline

Douglas RJ, Martin KA (2004) Neuronal circuits of the neocortex. Annu Rev Neurosci 27:419-451. CrossRef Medline

Engeland C, Mahoney C, Mohr E, Ilivitsky V, Knott VJ (2002) Acute nicotine effects on auditory sensory memory in tacrine-treated and nontreated patients with Alzheimer's disease: an event-related potential study. Pharmacol Biochem Behav 72:457-464. CrossRef Medline

Faber ES, Sah P (2003) Calcium-activated potassium channels: multiple contributions to neuronal function. Neuroscientist 9:181-194. CrossRef Medline

Fadel JR (2011) Regulation of cortical acetylcholine release: insights from in vivo microdialysis studies. Behav Brain Res 221:527-536. CrossRef Medline

Feldman H, Friston KJ (2010) Attention, uncertainty, and free-energy. Front Hum Neurosci 4:215. Medline

Felleman DJ, Van Essen DC (1991) Distributed hierarchical processing in the primate cerebral cortex. Cereb Cortex 1:1-47. CrossRef Medline

Friston K (2005) A theory of cortical responses. Philos Trans R Soc Lond B Biol Sci 360:815-836. CrossRef Medline 
Friston K (2008) Hierarchical models in the brain. PLoS Comput Biol 4: e1000211. CrossRef Medline

Friston K (2009) The free-energy principle: a rough guide to the brain? Trends Cogn Sci 13:293-301. CrossRef Medline

Friston K (2010) The free-energy principle: a unified brain theory? Nat Rev Neurosci 11:127-138. CrossRef Medline

Friston K, Kiebel S (2009) Predictive coding under the free-energy principle. Philos Trans R Soc Lond B Biol Sci 364:1211-1221. CrossRef Medline

Friston K, Harrison L, Daunizeau J, Kiebel S, Phillips C, Trujillo-Barreto N, Henson R, Flandin G, Mattout J (2008) Multiple sparse priors for the M/EEG inverse problem. Neuroimage 39:1104-1120. CrossRef Medline

Garrido MI, Kilner JM, Kiebel SJ, Friston KJ (2007a) Evoked brain responses are generated by feedback loops. Proc Natl Acad Sci U S A 104: 20961-20966. CrossRef Medline

Garrido MI, Kilner JM, Kiebel SJ, Stephan KE, Friston KJ (2007b) Dynamic causal modelling of evoked potentials: a reproducibility study. Neuroimage 36:571-580. CrossRef Medline

Garrido MI, Friston KJ, Kiebel SJ, Stephan KE, Baldeweg T, Kilner JM (2008) The functional anatomy of the MMN: a DCM study of the roving paradigm. Neuroimage 42:936-944. CrossRef Medline

Garrido MI, Kilner JM, Stephan KE, Friston KJ (2009a) The mismatch negativity: a review of underlying mechanisms. Clin Neurophysiol 120:453-463. CrossRef Medline

Garrido MI, Kilner JM, Kiebel SJ, Stephan KE, Baldeweg T, Friston KJ (2009b) Repetition suppression and plasticity in the human brain. Neuroimage 48:269-279. CrossRef Medline

Ghahramani Z, Beal MJ (2000) Variational inference for Bayesian mixtures of factor analysers. Adv Neural Inf Proc Syst 12:449-455.

Giovannini MG, Rakovska A, Benton RS, Pazzagli M, Bianchi L, Pepeu G (2001) Effects of novelty and habituation on acetylcholine, GABA, and glutamate release from the frontal cortex and hippocampus of freely moving rats. Neuroscience 106:43-53. CrossRef Medline

Haenschel C, Vernon DJ, Dwivedi P, Gruzelier JH, Baldeweg T (2005) Event-related brain potential correlates of human auditory sensory memory-trace formation. J Neurosci 25:10494-10501. CrossRef Medline

Hasselmo ME, McGaughy J (2004) High acetylcholine levels set circuit dynamics for attention and encoding and low acetylcholine levels set dynamics for consolidation. Prog Brain Res 145:207-231. CrossRef Medline

Hasselmo ME, Sarter M (2011) Modes and models of forebrain cholinergic neuromodulation of cognition. Neuropsychopharmacology 36:52-73. Medline

von Hemholtz H (1866) Die Lehre von den Gesichtswahrnehmungen. Handbuch der physiologische Optik III. Leipzig, Germany: Verlag von Leopold Voss.

Huang F, Lasseter KC, Janssens L, Verhaeghe T, Lau H, Zhao Q (2002) Pharmacokinetic and safety assessments of galantamine and risperidone after the two drugs are administered alone and together. J Clin Pharmacol 42:1341-1351. CrossRef Medline

Huang XY, Morielli AD, Peralta EG (1993) Tyrosine kinase-dependent suppression of a potassium channel by the $\mathrm{G}$ protein-coupled $\mathrm{m} 1$ muscarinic acetylcholine receptor. Cell 75:1145-1156. CrossRef Medline

Kiebel SJ, David O, Friston, KJ (2006) Dynamic causal modelling of evoked responses in EEG/MEG with lead field parameterization. Neuroimage 30:1273-1284. CrossRef Medline

Kiebel SJ, Garrido MI, Moran RJ, Friston KJ (2008) Dynamic causal modelling for EEG and MEG. Cogn Neurodyn 2:121-136. CrossRef Medline

Kiebel SJ, Daunizeau J, Friston KJ (2009) Perception and hierarchical dynamics. Front Neuroinform 3:20. Medline

Kilner JM, Kiebel SJ, Friston KJ (2005) Applications of random field theory to electrophysiology. Neurosci Lett 374:174-178. CrossRef Medline

Krause M, Pedarzani P (2000) A protein phosphatase is involved in the cholinergic suppression of the $\mathrm{Ca} 2+$-activated $\mathrm{K}+$ current s IAHP in hippocampal pyramidal neurons. Neuropharmacology 39:1274-1283. CrossRef Medline

Krnjevi K, Phillis J (1963) Acetylcholine-sensitive cells in the cerebral cortex. J Physiol 166:296-327. Medline
Lamme VA (1995) The neurophysiology of figure-ground segregation in primary visual cortex. J Neurosci 15:1605-1615. Medline

Liljenström H, Hasselmo ME (1995) Cholinergic modulation of cortical oscillatory dynamics. J Neurophysiol 74:288-297. Medline

Marr D (2010) Vision: a computational investigation into the human representation and processing of visual information. Cambridge MA: MIT.

Mattout J, Henson R, Friston K (2007) Canonical source reconstruction for MEG. Comput Intell Neurosci 2007:67613.

Miasnikov AA, Chen JC, Weinberger NM (2008) Specific auditory memory induced by nucleus basalis stimulation depends on intrinsic acetylcholine. Neurobiol Learn Mem 90:443-454. CrossRef Medline

Moran RJ, Jung F, Kumagai T, Endepols H, Graf R, Dolan RJ, Friston KJ, Stephan KE, Tittgemeyer M (2011) Dynamic causal models and physiological inference: a validation study using isoflurane anaesthesia in rodents. PloS One 6:e22790. CrossRef Medline

Moran RJ, Kiebel SJ, Stephan KE, Reilly RB, Daunizeau J, Friston KJ (2007) A neural mass model of spectral responses in electrophysiology. Neuroimage 37:706-720. CrossRef Medline

Moran RJ, Stephan KE, Kiebel SJ, Rombach N, O'Connor WT, Murphy KJ, Reilly RB, Friston KJ (2008) Bayesian estimation of synaptic physiology from the spectral responses of neural masses. Neuroimage 42:272-284. CrossRef Medline

Mumford D (1992) On the computational architecture of the neocortex. Biol Cybern 66:241-251. CrossRef Medline

Näätänen R, Astikainen P, Ruusuvirta T, Huotilainen M (2010) Automatic auditory intelligence: an expression of the sensory-cognitive core of cognitive processes. Brain Res Rev 64:123-136. CrossRef Medline

Nelson CL, Sarter M, Bruno JP (2005) Prefrontal cortical modulation of acetylcholine release in posterior parietal cortex. Neuroscience 132: 347-359. CrossRef Medline

Pepeu G, Giovannini MG (2004) Changes in acetylcholine extracellular levels during cognitive processes. Learn Mem 11:21-27. CrossRef Medline

Rao RP, Ballard DH (1999) Predictive coding in the visual cortex: a functional interpretation of some extra-classical receptive-field effects. Nat Neurosci 2:79-87. CrossRef Medline

Rodrigues S, Chizhov AV, Marten F, Terry JR (2010) Mappings between a macroscopic neural-mass model and a reduced conductance-based model. Biol Cybern 102:361-371. CrossRef Medline

Rokem A, Silver MA (2010) Cholinergic enhancement augments magnitude and specificity of visual perceptual learning in healthy humans. Curr Biol 20:1723-1728. CrossRef Medline

Samochocki M, Höffle A, Fehrenbacher A, Jostock R, Ludwig J, Christner C, Radina M, Zerlin M, Ullmer C, Pereira EF, Lübbert H, Albuquerque EX, Maelicke A (2003) Galantamine is an allosterically potentiating ligand of neuronal nicotinic but not of muscarinic acetylcholine receptors. J Pharmacol Exp Ther 305:1024-1036. CrossRef Medline

Sarter M, Parikh V, Howe WM (2009) Phasic acetylcholine release and the volume transmission hypothesis: time to move on. Nat Rev Neurosci 10:383-390. CrossRef Medline

Sarter M, Hasselmo ME, Bruno JP, Givens B (2005) Unraveling the attentional functions of cortical cholinergic inputs: interactions between signal-driven and cognitive modulation of signal detection. Brain Res Rev 48:98-111. CrossRef Medline

Thiel CM, Fink GR (2008) Effects of the cholinergic agonist nicotine on reorienting of visual spatial attention and top-down attentional control. Neuroscience 152:381-390. CrossRef Medline

Vossel S, Thiel CM, Fink GR (2008) Behavioral and neural effects of nicotine on visuospatial attentional reorienting in non-smoking subjects. Neuropsychopharmacology 33:731-738. Medline

Yu AJ, Dayan P (2002) Acetylcholine in cortical inference. Neural Netw 15: 719-730. CrossRef Medline

Yu A, Dayan P (2003) Expected and unexpected uncertainty: ACh and NE in the neocortex. Adv Neural Inf Proc Syst 15:173-180.

Yu AJ, Dayan P (2005) Uncertainty, neuromodulation, and attention. Neuron 46:681-692. CrossRef Medline

Zipser K, Lamme VA, Schiller PH (1996) Contextual modulation in primary visual cortex. J Neurosci 16:7376-7389. Medline 